[Contribution from the Chemical Laboratory, Maryland Academy of Sciences]

\title{
THE ELECTRICAL PRECIPITATION OF COLLOIDS
}

By Claude Haines Hali, JR.

Received February 21, 1922

\section{Introduction}

Bancroft ${ }^{1}$ suggests in his list of research problems in colloid chemistry that the precipitating action of high potential current on colloidal sus pensions in non-conducting liquid media should be investigated. There is apparently no literature upon this phase of the subject although a great deal of work has been done by Cottrell, ${ }^{2}$ Strong, ${ }^{3}$ and many others ${ }^{4,5}$ on the electrical precipitation of fogs and smokes.

The theory of smoke precipitation has been thoroughly worked out hy those mentioned, and is easily available so that it would serve no useful purpose to review it here. The case of the precipitation of sols is somewhat analogous to that of smokes although, of course, the viscosity of the medium is much greater and the electrical resistance of the medium is considerably higher. The optimum condition for the precipitation of smoke is a potential of about $5 \times 10^{4}$ volts, direct current, having the point or wire negative. ${ }^{4}$ The temperature is not important. ${ }^{3}$ On the basis of these data the precipitation of sols was attempted.

\section{Experimental}

The medium used was a very high grade transformer oil. ${ }^{6}$ It was rated by the manufacturer and also by a testing laboratory as having a resist. ance of $1.5 \times 10^{16}$ ohms per centimeter cube. The suspensions were prepared by several methods, the data for two of those used in the following tests being given as representative.

Test 4.-Two aluminum electrodes, connected to a $10^{4}$ volt transformer, were suspended in the oil and arranged so that they could be touched together and then separated a short distance. An arc was thus formed and maintained until the desired density was reached (about 10 minutes).

Test 1.-A sample of the oil was saturated with arsenic iodide ${ }^{7}$ and hydrogen sulfide passed in until the arsenic was completely precipitated.

${ }^{1}$ Bancroft, J. Ind. Eng. Chem., 13, 350 (1921).

2Cottrell, ibid., 3, 352 (1911); Smithsonian Rpt., 1913, 653.

${ }^{3}$ Strong, Proc. Am. Inst. Elec. Eng., 34, 387 (1915).

${ }^{4}$ Nesbit, ibid., 34, 405 (1915).

'Bradly, ibid., 34, 421 (1915).

'Supplied through the courtesy of Mr. E. P. Early of the Standard Oil Co.

7 Unpublished solubility tables. 
'This method makes a very dense and homogeneous sol, which will remain in suspension almost indefinitely. ${ }^{8}$

A number of forms of precipitation apparatus were tried out but most of them were found unsatisfactory owing to the great tendency of the current to break through. The form finally adopted was a vertical tube of Pyrex glass, $2 \mathrm{~cm}$. in diameter and $35 \mathrm{~cm}$. long, fitted with a circular platinum disk electrode at one end and a sharply pointed adjustable platinum wire at the other.

The coil used to supply the voltage was a $2 \times 10^{5}$ volt X-ray coil. For direct current work it was operated on 115 volts in connection with a battery of 3 electrolytic interrupters and a suitable primary condenser. A "point-to-plate" rectifier was inserted in the secondary circuit to suppress any "make" current. The secondary connections were carried directly, suitably suspended, to the electrodes of the tube and a test gap shunted across them. For alternating current tests the same coil was supplied with 80 volts from a small synchronous converter connected to the power lines. The interrupter, condenser and rectifier were, of course, omitted.

\begin{tabular}{|c|c|c|c|c|c|}
\hline \multirow[b]{2}{*}{ No. } & \multicolumn{4}{|c|}{ TABLE I } & \\
\hline & Suspension & $\begin{array}{c}\text { Nature of } \\
\text { charge on } \\
\text { point }\end{array}$ & $\begin{array}{l}\text { Secondary } \\
\text { potential } \\
\text { (Alternate } \\
\text { gap in } \mathrm{cm} . \text { ) }\end{array}$ & $\begin{array}{l}\text { Input } \\
\text { Kw. }\end{array}$ & $\begin{array}{l}\text { Time } \\
\text { Min. }\end{array}$ \\
\hline 1 & $\mathrm{As}_{2} \mathrm{~S}_{3}$ & $-D . C$. & 28 & 2 & 200 \\
\hline 2 & $\mathrm{HgS}$ & - D.C. & 20 & 2 & 70 \\
\hline 3 & $\mathrm{Fe}_{4}\left(\mathrm{FeCy}_{6}\right)_{3}$ & -D.C. & 20 & 2 & 60 \\
\hline 4 & $\mathrm{BaSO}_{4}$ & -D.C. & 20 & 2 & 120 \\
\hline 5 & $\mathrm{Al}$ & - D.C. & 28 & 2 & 200 \\
\hline 6 & $\mathrm{Cu}$ & -D.C. & 20 & 2 & 100 \\
\hline 7 & $\mathrm{~Pb}$ & -D.C. & 20 & 2 & 60 \\
\hline 8 & $\mathrm{Ag}$ & -D.C. & 20 & 2 & 120 \\
\hline 9 & $\mathrm{Fe}$ & - D.C. & 20 & 2 & 120 \\
\hline 10 & $\mathrm{As}_{2} \mathrm{~S}_{3}$ & +D.C. & 26 & 2 & 100 \\
\hline 11 & $\mathrm{HgS}$ & +D.C. & 20 & 2 & 120 \\
\hline 12 & $\mathrm{Al}$ & +D.C. & 25 & 2 & 130 \\
\hline 13 & $\mathrm{Cu}$ & + D.C. & 20 & 2 & 70 \\
\hline 14 & $A s_{2} S_{3}$ & A.C. & 16 & 1.5 & 200 \\
\hline 15 & $\mathrm{HgS}$ & A.C. & 12 & 1.2 & 90 \\
\hline 16 & $\mathrm{BaSO}_{4}$ & A.C. & 14 & 1.2 & 150 \\
\hline 17 & $\mathrm{Al}$ & A.C. & 10 & 1 & 100 \\
\hline 18 & $\mathrm{~Pb}$ & A.C. & 10 & 1 & 60 \\
\hline
\end{tabular}

The results were in every case the same. No matter how long the apparatus was run or what potentials were employed no sign of precipi-

${ }^{8}$ No difficulty was experienced from muddying of the oil when the sol was prepared by chemical methods. With the electrical method it is necessary to keep the current dersity very low (less than 0.01 ampere at the electrodes) if muddying is to be prevented. 
tation was observed. ${ }^{9}$ After each test the sol was removed from the precipitation tube and allowed to stand along with a sample of the same sol which had not been treated. No difference in the rate of settling could be noted although suspensions of widely differing coarseness were used, as may be seen from the fact that Test 6 settled in about 24 hours while Test 1 has not settled in over 6 months. The inevitable conclusion is that under the experimental conditions cited it is impossible to precipitate colloidal suspensions in non-conducting, liquid media by electrical means.

\section{Theoretical}

It is of interest to investigate the mathematical grounds for the failure of electrical precipitation in the case of sols. As Strong ${ }^{3}$ has shown in connection with smoke, the energy required for the precipitation of a colloid is $E=n\left(1 / 2 m V^{2}+F s\right)$ where $E$ is the energy in ergs per unit volume, $n$ the number of particles, $m$ the average mass of the particles, $V$ is the transverse velocity imparted to the particles, $F$ is the frictional resistance, and $s$ is the average distance traversed.

Stoke's law, which has been found to hold with considerable accuracy, gives $F=6 \pi \mu a d V$. Also $m=4 / 3 \pi d a^{3}$ and $s=1$, where $d$ is the density of the particles, $a$ the average radits, $\mu$ the viscosity of the medium, and $l$ the distance from point to plate for point electrodes, or the radius of the cylinder for cylindrical electrodes. Substituting, the formula becomes $E={ }^{2}{ }_{3} \pi d a^{3} V^{2}+3 \pi \mu a d l V$.

Roughly evaluating the relative constants as

$\begin{array}{lcc} & \text { Smokes } & \text { Sols } \\ \mu & 2.10^{-3} & 1 \\ a & 10^{-5} & 10^{-5} \\ l & 1 & 1 \\ d & 10^{-2} & 1 \\ V & V_{f} & V\end{array}$

transforming the above equation to a comparative form

$$
2 d_{s} a_{s}{ }^{3} V_{s}{ }^{2}+9 \mu_{s} a_{s} d_{s} l_{s} V_{s}=2 d_{j} a_{f}{ }^{3} V_{j}^{2}+9 \mu_{f} a_{f} d_{f} l_{s} V_{f}
$$

substituting the constants and solving for $V_{s}$ in terms of $V_{f}$.

$$
V_{s}=\frac{\left.-10^{-4} \pm \sqrt{10^{-8}+16 \cdot 10^{-15}\left(10^{-12} V_{f}^{2}+10^{-i} V_{f}\right.}\right)}{4 \cdot 10^{-15}}
$$

and expanding and simplifying (using the positive sign which gives the only root with a physical significance) we obtain,

$$
\begin{aligned}
& V_{s}=2 \cdot 10^{3}\left(10^{-i} V_{f}+10^{-17} V_{f^{2}}\right)+10^{-2}\left(10^{-7} V_{f}+10^{-17} V_{f}^{2}\right)+- \\
& V_{s}=2 \cdot 10^{-4} V_{f}+-+--
\end{aligned}
$$

${ }^{9}$ No corona effect was observed owing possibly to the opacity of the suspension, although the conditions in some of the tests must have been such that it could exist, particularly in Test 14. In Test 1 the electrodes were later drawn slowly together until spark-over occurred, no corona being observed. 
That is, that the velocity of precipitation for the same energy, applied to the particle, would be 20,000 times as great for smokes as for sols. This confirms the experimental evidence of the very slow precipitation.

\title{
Summary
}

The author has investigated the effect of high potentials upon colloidal suspensions in non-conducting liquids. There has been no precipitation detected in the range $10^{4}-2 \times 10^{5}$ volts, either alternating or direct current.

It is shown that the rate of precipitation would be $1 / 20000$ of that for smoke for the same energy applied to the particle.

The author wishes to acknowledge his gratitude to Mr. William Haggenbotham for the valuable assistance rendered him in the course of the investigation.

BALTIMORE, MARYLAND

[CONtribution from the Chemical, I,aboratory of Clark University, No. I, 11]

\section{THE EQUILIBRIUM IN LIQUID MIXTURES OF AMMONIA AND XYLENE}

\author{
By Charlfis A. Kraus and Endard H. Zeitfucis \\ Received March 8, 1922
}

\section{Introduction}

Franklin and Kraus have observed that mixtures of ammonia and metaxylene have an upper critical end-point ${ }^{t}$ a little below room temperatures. Since the vapor-pressure curves of only a few such systems have been studied thus far, ${ }^{2}$ it appeared worth while to investigate this system in some detail. In as much as the vapor pressure of xylene is low compared with that of ammonia, the total-pressure curves will differ little from the partial-pressure curves.

In the present investigation the vapor pressure of various liquid mixtures has been determined together with the composition of the liquid phases of the monovariant system. The latter data were determined in a separate series of experiments.

The critical end-point was determined in a special experiment by direct observation of the temperature at which the two phases become identical. For this purpose ammonia and metaxylene were sealed in a heavy walled

1 This is sometimes called the critical point of solution. Since such a point also occurs in the diphase system, it appears preferable to designate the critical point of the three-phase system as critical end-point, as has been suggested by Büchner.

2 The literature relating to systems of this type has been collected by Buichner in Part 2, Vol. II, of Roozeboom's "Die Heterogenen Gleichgewichte vom Standpunkte der Phasenlehre," and detailed references may accordingly be omitted here. 